\title{
Nivel de utilización de software epidemiológico en dos centros de salud primaria de la Provincia de Valdivia, 2004
}

\author{
MANUEL CÉSPEDES ${ }^{(1)}$, MARÍA CRISTINA TORRES ${ }^{(2)}$, FRANCISCO MARÍN ${ }^{(2)}$ Y JORGE GÓMEZ $^{(2)}$
}

\section{RESUMEN}

En el marco de la reforma de salud chilena, es imprescindible investigar la situación actual en la que se encuentran los centros de atención primaria con respecto al nivel de competencias, uso y valoración que el propio equipo de salud le otorga a las herramientas de vigilancia epidemiológica computacional existentes.

Este estudio es de tipo cuantitativo, transversal y descriptivo, desarrollado en el centro de atención primaria de la ciudad de Los Lagos, y en el Consultorio Externo Valdivia, durante el segundo semestre de 2004, a través de la aplicación de encuestas.

Los resultados muestran un bajo nivel de conocimientos y uso de software epidemiológico, con un 50,7\% que refiere no haber tenido a disposición algunos de estos programas. También se evidenció que el grupo que presenta mejor nivel de conocimiento informático es el de directivos, además de no encontrar diferencias estadísticamente significativas entre ambos centros de atención a pesar de su ubicación geográfica y disponibilidad de tecnología $(p<0,05)$.

El escaso conocimiento de software epidemiológico habla de la escasa y/o distante capacitación que se ha entregado a los equipos de salud, sumada a la falta de uso de estos programas, evidenciando, además la falta de prioridad de los niveles centrales.

Es altamente recomendable fomentar el uso de nuevas tecnologías en vigilancia epidemiológica, además de realizar un proceso de capacitación permanente, que sustente al desarrollo de los objetivos sanitarios que requieren obligadamente de estos procesos, para producir un impacto positivo en el perfil epidemiológico nacional.

Palabras Clave: vigilancia epidemiológica, epidemiología, software epidemiológico, capacitación.

\section{SUMMARY}

ASSESSMENT OF THE EXTENT OF EPIDEMIOLOGICAL SOFTWARE USE AT TWO PRIMARY CARE CENTERS OF THE VALDIVIA PROVINCE, DURING 2004.

Within the framework of the Chilean Health Reform, it is critical to assess the current situation of primary care centers specifically concerning the state of human skills, the use of the available computational epidemiological surveillance tools and the appraisal of such tools by the health team.

The present study is quantitative, cross-sectional and descriptive in nature, and it has been developed at the Primary Care Center of the city of Los Lagos, and at the Valdivia Outpatient Clinic, during the second semester of 2004, using a survey method.

\footnotetext{
(1) Matrón. Hospital Base Valdivia. manuelcespedes@uach.cl

(2) Académico. Instituto de Salud Pública. U. Austral
} 
The results showed a low level of knowledge and a poor use of epidemiological software, with $50.7 \%$ expressing not having some of such programs available. The best levels of informatics knowledge were evidenced among the management. Additionally, there were no statistically significative differences between both care centers in spite of their dissimilar geographic location and technology availability $(p<0.05)$.

The poor knowledge about epidemiological software evidences the limited and distant training that has been provided to health teams, a fact that adds up to a deficient use of such programs, reflecting a lack of priority at the central levels.

It is highly advisable to encourage the use of novel technologies in epidemiological surveillance, together with a continuing education process, to support the development of health goals for which such processes are mandatory, in order to result in a positive impact on the national epidemiological profile.

Keywords: epidemiological surveillance, epidemiology, epidemiological software, training.

\section{INTRODUCCIÓN}

Los profesionales de la salud han debido sortear una serie de desafíos, para superar las enfermedades que se han suscitado según cada época. Para superar estas brechas se han utilizado herramientas diferentes, pero todas con un solo fin: mejorar la toma de decisiones para entregar una mejor atención y dar respuestas a las reales necesidades de la población en los distintos niveles de salud.

Hoy la informática ha llegado a ser considerada como "imprescindible" para la evolución de las empresas e instituciones. La versatilidad que ofrece y sus bajos costos que caracterizan la puesta en marcha de esta herramienta la han hecho identificable, en mayor o menor medida, en prácticamente todas las instituciones de salud.

El nivel de explotación de esta herramienta, está mediado por múltiples factores, entre éstos se encuentran la disponibilidad de recursos, la capacitación del personal y las políticas que estimulan la puesta en marcha de estas iniciativas, que hoy no dejan de ser innovadoras a nivel local.

En el marco de la reforma de salud chilena, se hace necesario vigilar activa y precisamente cada evento sanitario producido en cualquier nivel de atención, sobretodo en eventos relacionados directamente con los Objetivos Sanitarios $^{1}$. De esta manera, podemos dar respuestas a una serie de interrogantes en tiempos muy cortos, lo que junto con mejorar la calidad de atención sirve para disminuir costos. Todo esto depende de la capacidad del personal para dar pie a la innovación, y del estímulo que den las instituciones, para la puesta en marcha y desarrollo de estos sistemas.

Estos procesos pueden ser familiares para los grandes centros de atención, que ya se han visto en la necesidad de realizar su propia vigilancia epidemiológica. Pero si nos situamos en los pequeños consultorios y hospitales, podemos observar empíricamente el escaso uso de sistemas informáticos que apoyen la toma de decisiones, debido a que hasta el momento han podido sortear con mediano éxito el conteo de sus eventos, simplemente a través de sistemas manuales.

Por otro lado, no podemos olvidar que si bien, la epidemiología en sus inicios sirvió para la cuantificación de enfermedades transmisibles y sus "epidemias", hoy en día, esta ciencia ha pasado de este tipo de enfermedades a la vigilancia de factores de riesgo ${ }^{2}$, relacionados principalmente, con la aparición de enfermedades crónicas como lo son la hipertensión arterial y la diabetes.

El cuestionamiento final pasa a ser, entonces, si los equipos de salud de los pequeños centros de atención, están realmente capacitados para realizar su propia vigilancia epidemiológica, a través del uso de herramientas informáticas, que van desde el manejo básico de un computador, 
hasta el uso de bases de datos, paquetes estadísticos y sistemas de información geográfica.

\section{MATERIAL Y MÉTODO}

Este estudio es de tipo cuantitativo, descriptivo y transversal, desarrollado en las ciudades de Valdivia, capital de la provincia del mismo nombre, y en Los Lagos, ciudad ubicada a $50 \mathrm{~km}$. de Valdivia, ambas ubicadas en la X Región de Los Lagos.

La población en estudio abarca a los equipos de salud pertenecientes al Consultorio Externo Valdivia y al Consultorio adosado de la ciudad de Los Lagos, durante el segundo semestre de 2004. Se entiende por equipo al grupo de: administrativos, auxiliares de enfermería, profesionales, encargados de programas y directivos.

La selección de la muestra se realizó, al interior de cada consultorio, mediante muestreo aleatorio simple, con un nivel de confianza de $95 \%$ y un índice de precisión del 7\%. Correspondientes a 41 personas en el Consultorio Externo Valdivia y 22 personas en el Consultorio adosado de Los Lagos.

La recolección de la información se realizó mediante una encuesta auto aplicada, diseñada por el propio investigador; testeada previamente en un centro de similares características; debido a la no existencia de instrumentos para medir las competencias requeridas.

Se resumen los datos mediante media aritmética y desviación estándar (DE), y el resumen de variables cualitativas mediante proporciones. La significación estadística se determina mediante la dócima de asociación ji cuadrado con un nivel de significancia de 5\%. Estos procedimientos se realizaron mediante la utilización del programa estadístico Epi Info 2002®.

\section{RESULTADOS}

Distribución del personal según conocimientos sobre el uso de herramientas computacionales

Con respecto al uso del Sistema Operativo Windows, el 54,1\% de los encuestados refiere utilizar "programas que conoce", representando la mayor frecuencia en ambos consultorios. En el grupo de directivos, la misma respuesta se eleva a un $64,3 \%$. No se observan diferencias estadísticamente significativas entre ambos consultorios $(p=0,82)$.

El porcentaje de encuestados considerados como analfabetos en Internet (27,9\%), es decir, que nunca han tenido acceso o no saben utilizarlo, es similar al nivel de analfabetos computacionales $(29,5 \%)$. No se observaron diferencias estadísticamente significativas entre ambos consultorios $(\mathrm{p}=0,13)$.

La distribución del grupo de encuestados que saben utilizar el correo electrónico, según grupo etario, concentra la mayor frecuencia entre los 25 y 34 años. El promedio de edad del personal que utiliza esta herramienta alcanza $\operatorname{los} 38,23$ años $(\mathrm{DE}=10,3)$.

\section{Distribución del personal encuestado según conocimientos sobre software epidemiológico}

El 65,5\% de los encuestados refiere no haber recibido capacitación sobre el uso de software de vigilancia epidemiológica. Este valor disminuye a un 50\% al contrastar sólo el grupo de directivos. No se encontraron diferencias estadísticamente significativas entre ambos consultorios $(\mathrm{p}=0,91)$.

Con respecto al grupo de directivos, y su nivel de conocimientos acerca del uso de herramientas de vigilancia epidemiológica, un $42,9 \%$ refiere que sus conocimientos son nulos, cifra superior confrontando la misma respuesta con el grupo general, seguidos por el 35,7\% con conocimientos de nivel medio, siendo mayor al total general. Cabe destacar que ningún encuestado del grupo directivo consideró que sus conocimientos son de tipo alto. No se encontraron diferencias estadísticamente significativa al confrontar ambos consultorios $(p=0,4)$.

Con respecto al uso específico de software de vigilancia epidemiológica, el más conocido es Epi Info $\AA(23,8 \%)$. Existe diferencia estadísticamente significativa entre el grupo de directivos $(50,0 \%)$ y el grupo en general $(21,4 \%)$ 
Tabla 1. Distribución de software utilizado por personal de salud.

Según establecimiento. Año 2004

\begin{tabular}{|l|r|r|r|r|r|r|}
\hline \multirow{2}{*}{$\begin{array}{l}\text { Software de vigilancia } \\
\text { utilizado }\end{array}$} & \multicolumn{2}{|c|}{ Los Lagos } & \multicolumn{2}{c|}{ CEV } & \multicolumn{2}{c|}{ TOTAL } \\
\hline & $\mathbf{N}^{\mathbf{0}}$ & $\mathbf{\%}$ & $\mathbf{N}^{\mathbf{0}}$ & $\mathbf{9}$ & $\mathbf{N}^{\mathbf{0}}$ & \% \\
\hline Citoexpert & 2 & 9,1 & 3 & 7,3 & 6 & 8,9 \\
\hline Epi Info & 6 & 27,3 & 10 & 24,3 & 16 & 23,8 \\
\hline Epi Vigil & 0 & 0,0 & 1 & 2,4 & 1 & 1,5 \\
\hline Inscrito & 1 & 4,5 & 3 & 7,3 & 4 & 5,9 \\
\hline Módulo atención & 0 & 0,0 & 2 & 4,8 & 2 & 2,9 \\
\hline SIP & 1 & 4,5 & 0 & 0 & 1 & 1,5 \\
\hline SIG & 0 & 0,0 & 0 & 0 & 0 & 0 \\
\hline Otro & 2 & 9,1 & 1 & 2,4 & 3 & 4,4 \\
\hline Ninguno & 10 & 45,5 & 21 & 51,2 & 34 & 50,7 \\
\hline TOTAL & $\mathbf{2 2}$ & $\mathbf{1 0 0 , 0}$ & $\mathbf{4 1}$ & $\mathbf{1 0 0}$ & $\mathbf{6 7}$ & $\mathbf{1 0 0}$ \\
\hline
\end{tabular}

Tabla 2. Distribución de tipos de tareas realizadas en software de vigilancia.

Según establecimiento. Año 2004

\begin{tabular}{|l|r|r|r|r|r|r|}
\hline $\begin{array}{l}\text { Tipo de utilización de } \\
\text { software de vigilancia }\end{array}$ & \multicolumn{2}{|c|}{ Los Lagos } & \multicolumn{2}{c|}{ CEV } & \multicolumn{2}{c|}{ TOTAL } \\
\hline & $\mathbf{N}^{\mathbf{0}}$ & $\mathbf{1}$ & $\mathbf{N}^{\mathbf{0}}$ & $\mathbf{0}$ & $\mathbf{N}^{\mathbf{0}}$ & $\%$ \\
\hline No utiliza NS/NR & 14 & 63,6 & 28 & 75,7 & 43 & 70,5 \\
\hline Ingreso de datos & 1 & 4,5 & 1 & 2,7 & 2 & 3,3 \\
\hline Consulta de datos & 5 & 22,7 & 3 & 8,1 & 9 & 14,8 \\
\hline $\begin{array}{l}\text { Generación de tablas } \\
\text { de contingencia } \\
\text { y/o gráficos }\end{array}$ & 0 & & & & & \\
\hline $\begin{array}{l}\text { Cálculo de fórmulas } \\
\text { tasas, porcentajes, } \\
\text { nivel de confianza, etc) }\end{array}$ & 2 & 0 & 3 & 8,1 & 3 & 4,9 \\
\hline TOTAL & $\mathbf{2 2}$ & $\mathbf{1 0 0}$ & $\mathbf{3 7}$ & $\mathbf{1 0 0}$ & $\mathbf{6 1}$ & $\mathbf{1 0 0}$ \\
\hline
\end{tabular}

con respecto a haber tenido a disposición y utilizado este software $(p=0,03)$, el resto de los software de vigilancia utilizados se especifican en la Tabla 1. Las tareas para las que se utilizan estos programas se resumen en la Tabla 2.

\section{DISCUSIÓN}

A pesar de que el sector primario de salud, puede ser considerado, un prestador de servicios convencionales, se puede evidenciar que existe un nivel aceptable de conocimientos en cuanto al uso de tecnologías computacionales a nivel general. Este conocimiento concuerda con Oinas y Malecki ${ }^{3}$, quienes postulan que la producción eficiente es cada vez más dependiente de tecnologías modernas, aunque se trate de servicios convencionales.

En cuanto al nivel de conocimientos de software epidemiológico, se mantiene la 
tendencia del escaso apoyo a estas iniciativas, enunciadas por Boggiano ${ }^{4}$, evidenciado por el bajo nivel de conocimientos detectados en este estudio, a pesar que desde la década pasada se está hablando de la importancia de la automatización de procesos y de su ahorro por sobre los procesos en forma manual ${ }^{5}$. Lo que está sustentado, además, por la pérdida de tiempo al realizar los registros en forma manual ${ }^{6}$.

Este bajo conocimiento, puede estar explicado, al menos, en uno de los tres puntos débiles de la capacitación, propuestos por Davini, este es: la alta rotatividad, movilidad e inestabilidad del personal de los servicios?

Otro causante es, la imposición de estos sistemas con una deficiente intervención del personal en su desarrollo, lo que contrasta con las recomendaciones de Dean ${ }^{5}$, quien generó el sistema Epi Info, en conjunto con personal que lo utiliza en Estados Unidos.

El escaso contacto que tienen los epidemiólogos, al representar un recurso escaso, también representa una piedra de tope a la hora de mejorar el nivel de conocimientos con respecto al uso de sistemas de vigilancia epidemiológica computacional. El aumento de estos profesionales, según Dussault ${ }^{8}$, puede mejorar esta brecha.

Es necesario dar un giro a la concepción clásica o antigua de la epidemiología, la cual se refería a la vigilancia de enfermedades infectocontagiosas, que todavía se sigue utilizando, pero no representa hoy las prioridades de los centros de salud. El cambio orienta a la vigilancia de factores de riesgo ${ }^{9}$.

Otra forma de mejorar o estimular el uso de estos sistemas, es la estrecha relación que se generará entre las herramientas informáticas y el modelo de evaluación de los "Objetivos Sanitarios para la Década" propuestos por el Ministerio de Salud ${ }^{1}$. Estos objetivos generan una serie de indicadores a ser medidos y analizados, estimulando además el vuelco hacia la vigilancia de enfermedades crónicas en la epidemiología.

En cuanto a la capacitación sobre herramientas informáticas, el personal concuerda con Davini ${ }^{7}$, al preferir una institución educacional como la entidad idónea para la generación de vigilancia epidemiológica. También se estima que será de gran apoyo la creación de alianzas estratégicas con la Autoridad Sanitaria (Seremi), para establecer coordinadamente una agenda de capacitación para la generación de sistemas de vigilancia, en pos de sus objetivos sanitarios.

Si profundizamos en el tema de los Sistemas de Información Geográfica, se puede decir que el nulo contacto de los trabajadores encuestados con este tipo de herramienta, es debido a que su uso en Salud Pública es reciente ${ }^{10}$, aunque es preocupante que, a pesar de ser Chile uno de los países pioneros en América Latina, junto con Guatemala, México y Cuba, se posea un conocimiento y contacto tan bajos con estas herramientas ${ }^{11}$ que se están tratando de implementar a nivel ministerial.

A pesar de que el servicio de salud ha impuesto sistemas de vigilancia en entornos locales (Citoexpert, Sistema Informático Perinatal, etc). Se mantiene el correlato entre la importancia política de la capacitación, y los recursos destinados para su desarrollo ${ }^{7}$. Se puede observar que de vez en cuando se realizan capacitaciones, aunque carentes de continuidad, sin mencionar la poca actualización de los programas utilizados a la nueva tecnología existente.

La relación de conocimientos evidenciados por los directivos, en contraste al resto del equipo, concuerda con el enunciado de Maldonado ${ }^{12}$, que identifica a la Epidemiología como el sustrato para la toma de decisiones, decisiones que deben ser tomadas por la planta de directivos de los centros de salud. Además de la existencia de una reforma de salud, que promueve el desarrollo de establecimientos autogestionados, con compromisos de gestión elaborados en relación a objetivos epidemiológicos claros y precisos.

Al contrastar ambos centros de salud, no se observan diferencias significativas, tomando en cuenta que sí existen diferencias geográficas en cuanto a distancias de polos de desarrollo, como pueden ser centros universitarios o el servicio 
de salud. Esta comparación dista de la opinión vertida por Rallet y Torre ${ }^{13}$, quienes refieren la importancia de la proximidad geográfica en la capacitación. Esto puede ser explicado por el crecimiento exponencial de las vías de comunicaciones y telecomunicaciones. Cabe mencionar que estos autores refutan el argumento de que las tecnologías de la información habrían reducido la importancia de la proximidad, entonces, esto significa que nos encontramos actualmente en un proceso de transición en cuanto a las tecnologías utilizadas en la capacitación.

Para concluir, los centros de salud no están actualmente capacitados para generar informáticamente sus propios datos epidemiológicos, los conocimientos aún son muy bajos en esta área, generando preocupación debido a la proximidad de la autogestión en redes, que necesita de la generación de propios indicadores de salud. Por lo que es altamente recomendable realizar una capacitación permanente, debido a que la discontinuidad genera la pérdida de conocimiento, sumando personal experto que dé soluciones rápidas para no perder la mantención de los procesos y, por último, es recomendable fomentar la investigación y promoción de esta área, que por el momento ha sido poco explorada en nuestro país, en contraste a países con más experiencia que llevan más de dos décadas dedicados a esta área ${ }^{14}$.

\section{REFERENCIAS}

1. MINSAL. 2000. Objetivos sanitarios para la década 2000-2010

2. MORAVIA, A. 1996. From Diseases to the Survillance of Risk Factors. Am. J. Public Health.. 86 (5): 625-626.
3. OINAS, P. \& E. Malecki 1999. "Spatial innovation systems". Malecki, E. \& P. Oinas, Making connections, technological learning and regional economic change. Aldershot: Ashgate.

4. BOGGIANO, H. Informática en un centro de atención primaria de salud (APS) del sub-sector público: Tecnología apropiada? Informática Médica $\mathrm{N}^{\circ} 2$. Argentina.(disponible en:

http://www.informaticamedica.org.ar/numero2/aps $2 . h t m$ Consultado el: 15/05/04).

5. DEAN AG, FAGAN RF, PANTER-CONNAH BJ. 1994."Computerizing Public Health Surveillance Systems". Principles and Practice of Public Health Surveillance. Oxford University Press. 201-217.

6. TORRES M.C. s.f. Relación costo efectividad. Control Prenatal en establecimientos seleccionados. Región de Los Lagos.

7. DAVINI M. 2002. Capacitación del personal de los Servicios de Salud. Proyectos relacionados con la reforma sectorial. OPS-OMS. Quito - Ecuador.

8. DUSSAULT G. 1995. "Epidemiology and Health Services Management". Pan American Health Organization. Epidemiological Bulletin. Vol. 16, $\mathrm{N}^{\circ} 2$.

9. MORAVIA, A. 1996. From Diseases to the Survillance of Risk Factors. Am. J. Public Health.. 86 (5): 625-626.

10. OPS. 1996. Uso de sistemas de información geográfica en epidemiología (SIG-Epi). Vol. 17, No. 1.

11. MINSAL. Foro SIGepi-Chile Experiencias y proyectos de SIG en Salud en Chile (disponible en: http://epi.minsal.cl/SigEpi/chile.htm. Consultado el: 21/05/05).

12. MALDONADO, O. 1997. Desarrollo de Aplicación Computacional para Vigilancia en Salud Pública en el Servicio de Salud Atacama. Tesis Magíster en Salud Pública Mención Epidemiología. Universidad de Chile. Facultad de Medicina. 215 p.

13. RALLET, A. \& A. TORRE 1999. "Is geographical proximity necessary in the innovation networks in the era of global economy?" GeoJournal, 49: 373380 .

14. VALLERÓN A. y cols. 1986. A computer network for the Survillance of Communicable Diseases: the French Experiment. Am. J. Public Healh. 76 (11): 1289-1292.

Recepción : jun. 2006

Aprobación : 9 ene. 2007

Usted puede comentar éste y otros artículos publicados en la Revista Chilena de Salud Pública, enviando un correo electrónico a revistasp@med.uchile.cl 